\title{
Asymmetric Addition of Allylsilanes to Aldehydes - A Cr/Photoredox Dual Catalytic Approach Complementing the Hosomi-Sakurai Reaction
}

Felix Schäfers, § Subhabrata Dutta,§ Roman Kleinmans, Christian Mück-Lichtenfeld and Frank Glorius*

Organisch-Chemisches Institut, Westfälische Wilhelms-Universität Münster, Corrensstraße 36, 48149 Münster

*Corresponding author: glorius@uni-muenster.de

$\S$ Authors contributed equally.

Abstract: The allylation of aldehydes is a fundamental transformation in synthetic organic chemistry. Among the multitude of available reagents, especially allylsilanes have been established as preferred allyl source. As initially reported by Hosomi \& Sakurai, these non-toxic and highly stable reagents add to carbonyls via an open transition state upon Lewis acid activation.

Herein, we report a general strategy to access a variety of valuable homoallylic alcohols in opposite chemo- and diastereoselectivity to the established Hosomi-Sakurai conditions by switching to photocatalytic activation in combination with a closed transition state (Chromium catalysis). Moreover, this dual catalytic approach displays a straightforward way to introduce excellent levels of enantioselectivity and its mild conditions allow for a broad substrate scope including chiral boronsubstituted products as a highlight.

To emphasize the synthetic utility, our method was applied as the key step in the synthesis of a bioactive compound and in the late-stage functionalization of steroid derivatives. Detailed mechanistic studies and DFT calculations hint towards an unprecedented photo-initiated chain being operative. 
Introduction: Homoallylic alcohols are highly valuable building blocks in natural product synthesis, drug discovery and materials science. ${ }^{1}$ They are a common motif in numerous bioactive molecules ${ }^{2}$ (Figure 1) and the dense accumulation of functionality allows them to serve as synthetic linchpins towards several other related targets. Consequently, accessing those structures has received enormous attention by the synthetic community and due to the presence of a chiral center, it always has been a benchmark reaction for enantioselective transformations. ${ }^{3}$

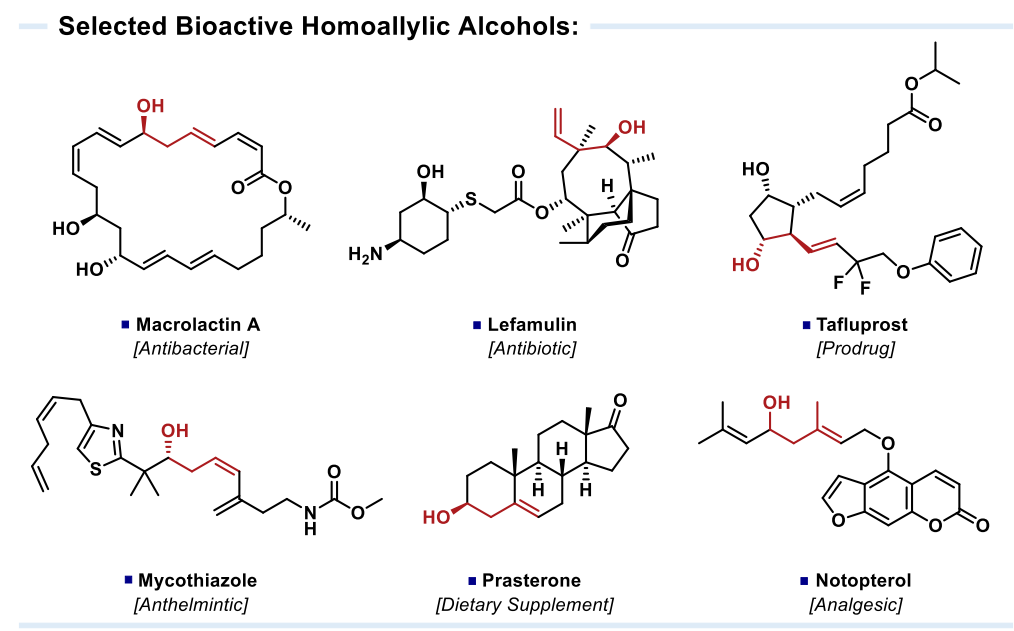

Figure 1. Selected examples of important homoallylic alcohols.

Arguably the most straightforward disconnection to generate this motif is the allylation of carbonyl compounds. Fundamental research on this valuable $\mathrm{C}-\mathrm{C}$ bond formation was performed early on and led to the development of a variety of allylic organometallic reagents, ${ }^{4}$ utilizing (semi)metals such as $\mathrm{Zn}$, In, B, Sn, Mn, Sb, Mg, Bi, Cr or Al. ${ }^{5}$ Later, focus predominantly switched towards the development of catalytic methods, ${ }^{6}$ avoiding the (over)stoichiometric use of these often toxic and/or expensive reagents while significantly facilitating access to enantioenriched products. As a result, especially the catalytic use of chiral metal complexes became state of the art as a convenient method to exert stereocontrol in allylation reactions. ${ }^{7}$ Important contributions include for example the utilization of allylic acetates, ${ }^{8,9}$ allylic halides, ${ }^{10,11}$ allenes ${ }^{12-14}$ or allylic $\mathrm{C}-\mathrm{H}$ functionalization. ${ }^{15}$

Among this variety of reagents, allylsilanes turned out to be an ideal allyl source, ${ }^{16}$ standing out due to their abundance and low toxicity. In addition, they show a remarkably high stability, which simplifies their synthesis, purification and storage and results in a huge library of functionalized allylsilane derivatives being easily accessible. ${ }^{17,18}$ However, once combined with activated electrophiles, allylsilanes show a high level of reactivity, as intermediately formed charges are stabilized by the $\beta$-silicon effect. ${ }^{19}$ 
Recognizing this huge potential, Hosomi \& Sakurai initially reported the allylation of carbonyl compounds with allyltrimethylsilane in 1976 (Figure 2a). ${ }^{20}$ While early reports were focused on strong Lewis acid activators (e.g. $\mathrm{TiCl}_{4}, \mathrm{SnCl}_{4}, \mathrm{AlCl}_{3}$ or $\left.\mathrm{BF}_{3} \cdot \mathrm{Et}_{2} \mathrm{O}\right)^{21}$ to increase the electrophilicity of the carbonyl compound, the activation of the allylsilane by fluoride has been reported as well, but typically leads to the loss of regioselectivity. ${ }^{21-23}$ In both cases, the key nucleophilic addition step was found to proceed via an open transition state. ${ }^{24}$

The necessity of electronic activation and the open transition state determine the inherent characteristics and limitations of this approach. First of all, the addition of a rather harsh activating reagent reduces the functional group tolerance significantly. ${ }^{25}$ Moreover, allylsilanes bearing electron-withdrawing groups often show diminished reactivity as they are not electron-rich enough to undergo an efficient nucleophilic attack. Electron-rich allylsilanes however, not only readily react with aldehydes, but also with different carbon electrophiles such as imines, acetals, ketones or epoxides. ${ }^{26}$ While this high level of reactivity renders the method quite versatile, the lack of chemoselectivity can also turn out as a drawback, for instance in late-stage functionalization.

The selectivity of branched Hosomi-Sakurai products $^{24}$ is determined by the anti-periplanar arrangement of reactants leading to the selective formation of the syn-product. At the same time the open transition state impedes the introduction of enantioselectivity. Nevertheless, impressive progress has been made tackling this challenging problem (Figure $2 \mathrm{~b}$ ). After the utilization of chiral, tartratemodified allylsilanes only showed limited success, ${ }^{27}$ a variety of important contributions proved catalytic chiral Lewis acid ${ }^{28,29}$ or Lewis base ${ }^{30-32}$ strategies to be better suited. However, the non-selective silylium background reaction was found to present a problem under Lewis acidic conditions leading to the necessity of high catalyst loadings. ${ }^{33}$ Lewis base strategies on the other hand rely on the use of highly reactive trichloroallylsilanes and thus often show limited substrate scope and low functional group tolerance. A promising but underrepresented alternative displays the utilization of transition metals $\left(\mathrm{Ag},{ }^{34}\right.$ $\mathrm{Cu}^{35}$ ) in combination with fluoride activation and chiral phosphine ligands, although the exact mode of activation as well as its generality has not been fully explored yet. Recently, very impressive results have been obtained by the use of chiral Brønsted acids as organocatalysts. ${ }^{3}$ This protocol utilizes highly elegant but rather complex catalyst structures, with different derivatives thereof being tailormade for either aliphatic or aromatic aldehydes. In addition, the reaction has to be performed at $-78^{\circ} \mathrm{C}$ for up to 
eight days and neither the reaction of substituted allylsilanes nor the broad tolerance of functional groups was shown.

In summary, a general and user-friendly method displaying a broad substrate scope, excellent enantioselectivity, high functional group tolerance and opposite chemo- and diastereoselectivity to these previous approaches would be highly desirable.

a. Hosomi-Sakurai Allylation:
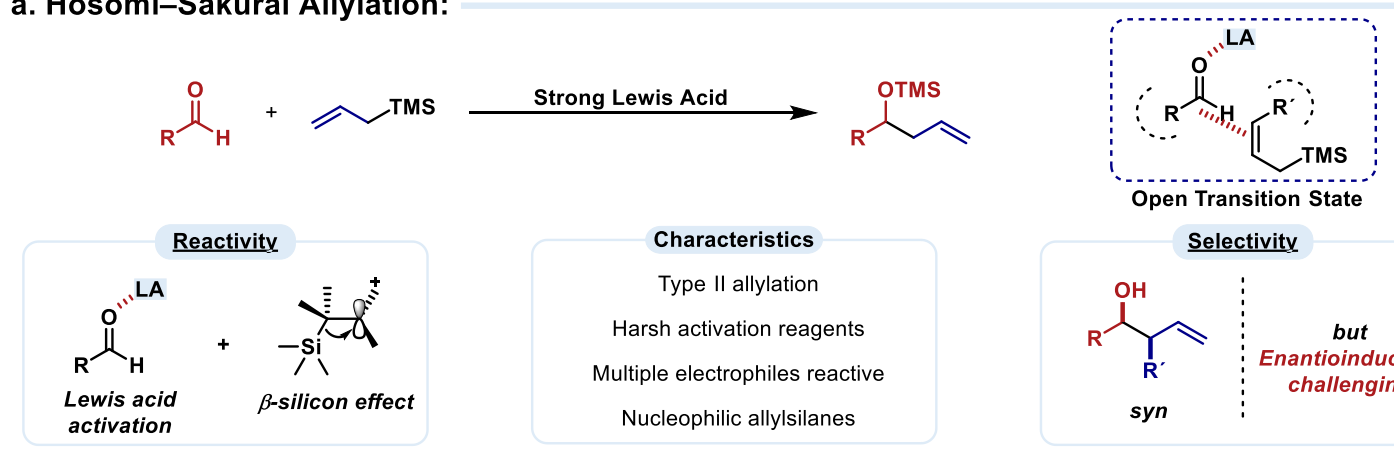

. Enantioselective Approaches:

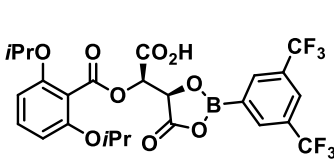

Chiral Lewis acid

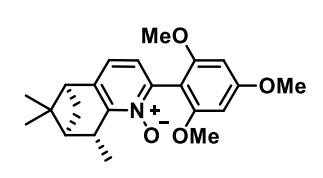

Chiral Lewis base

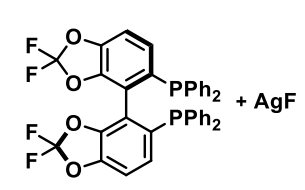

Transition metal + fluoride
Open Transition State

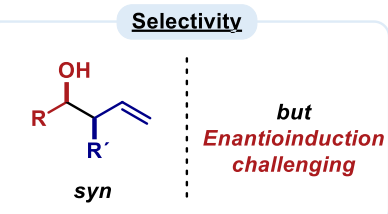

c. New Dual Catalytic Approach:

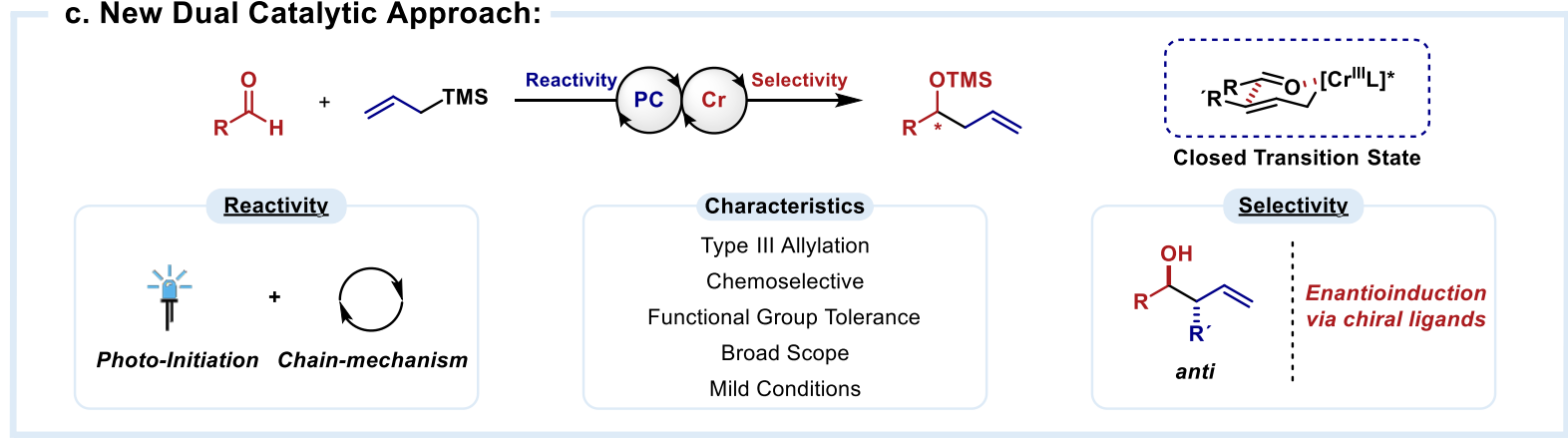

Figure 2. Strategies towards the synthesis of homoallylic alcohols utilizing allylsilanes.

Therefore, we questioned if one could make use of the advantages of allylsilanes as ideal allyl source, but bypass the inherent limitations of the reported ionic pathways. A solely radical strategy however would likely complement the ionic activation, but lack the required selectivity. This reactivity/selectivity issue has led to the development of a powerful strategy combining the high reactivity of radicals with the selectivity of organometallic intermediates, called dual photoredox catalysis. ${ }^{36}$ Looking at the HosomiSakurai reaction from a dual catalytic perspective (Figure 2c), a mild photocatalytic activation would allow for high flexibility in regards to the electronic nature of the allylsilane combined with a high functional group tolerance resulting from the avoidance of harsh activation reagents. Searching for the 
fitting metal, we chose chromium, a cheap and surprisingly non-toxic ${ }^{37} 3 \mathrm{~d}$ metal for three reasons. First, allyl-chromium species have been shown to be compatible with dual catalytic conditions ${ }^{38,39}$ and react via a closed Zimmerman-Traxler transition state. This would result in the formation of the antidiastereomer, thus displaying opposite selectivity to the Hosomi-Sakurai conditions. Secondly, in addition to their low basicity, allyl-chromium species show high chemoselectivity towards aldehydes even in presence of other electrophiles. Lastly, the in-situ generation of chiral chromium complexes by ligand addition presents an optimum way to introduce enantioselectivity. To the best of our knowledge such a dual catalytic platform to couple allylsilanes with aldehydes mediated by visible light has not been developed so far (Figure 2c).

Reaction Optimization: Bearing the literature background on organochromium-species ${ }^{38,40-43}$ and the SET-oxidation of allylsilanes ${ }^{44}$ in mind, a dual catalytic approach seemed feasible. Indeed, when irradiating a mixture of the organic dye 9-mesityl-10-methylacridinium tetrafluoroborate together with allyltrimethylsilane, $\mathrm{CrCl}_{2}$ and aldehyde 1 with blue LEDs in acetonitrile, product formation was observed, but the reaction quickly decolorized before full conversion was achieved. This indicated that the stability of the photocatalyst plays a crucial role and after switching to the sterically shielded acridinium derived PC2, developed by our group, ${ }^{45}$ a significantly improved performance was observed. To ensure tolerance of sterically and electronically demanding substituents, a more challenging allylsilane was searched as model substrate. Considering the relevance of boron compounds particularly in pharmaceutical and agrochemical method development, ${ }^{46}$ bis(pinacolato)diboron (Bpin) substituted allylsilane $\mathbf{2}$ was chosen. Having identified the optimum photocatalyst and the model substrate, several established chiral ligands were tested (Table 1, L 1-6). Fortunately, bisoxazoline ligand 1, pioneered in chromium catalysis by Kanai, ${ }^{39}$ gave excellent results. Sulfonamide ligand $\mathbf{6}$ however gave an improved yield, but diminished selectivity (entry 13). After final optimization, the ideal reaction conditions were identified (Table 1), giving the homoallylic product 3 in $75 \%$ isolated yield and an excellent enantiomeric ratio (er > 99:1). 
Table 1. Optimized conditions and deviation table.

Deviation from Std. Conditions

Control reactions revealed that the reaction does not proceed without chromium, light or photocatalyst (entries $2-4)$. Instead it was found that the reaction still works effectively when only slight excess of the allylsilane is used (entry 7). Interestingly, the reaction was observed to reach full conversion unexpectedly fast $(\sim 4 \mathrm{~h}$ without and $\sim 12 \mathrm{~h}$ with ligand). To ensure reproducibility and scalability, a sensitivity assessment was conducted (Figure 3 ). The results show, that the careful exclusion of water and oxygen is critical for the reaction to proceed and yield and enantioselectivity can both be transferred excellently to applications on larger scale $(2 \mathrm{mmol})$.

Substrate Scope: A variety of different coupling partners was tested to evaluate the generality and functional group tolerance of our asymmetric protocol (Figure 3). The TMS-protected homoallylic alcohols obtained can either be isolated as such or quantitatively deprotected during the work-up (see SI). Without ligand typically higher reactivity is observed and the racemates are obtained in shorter reaction time and often higher yield. 


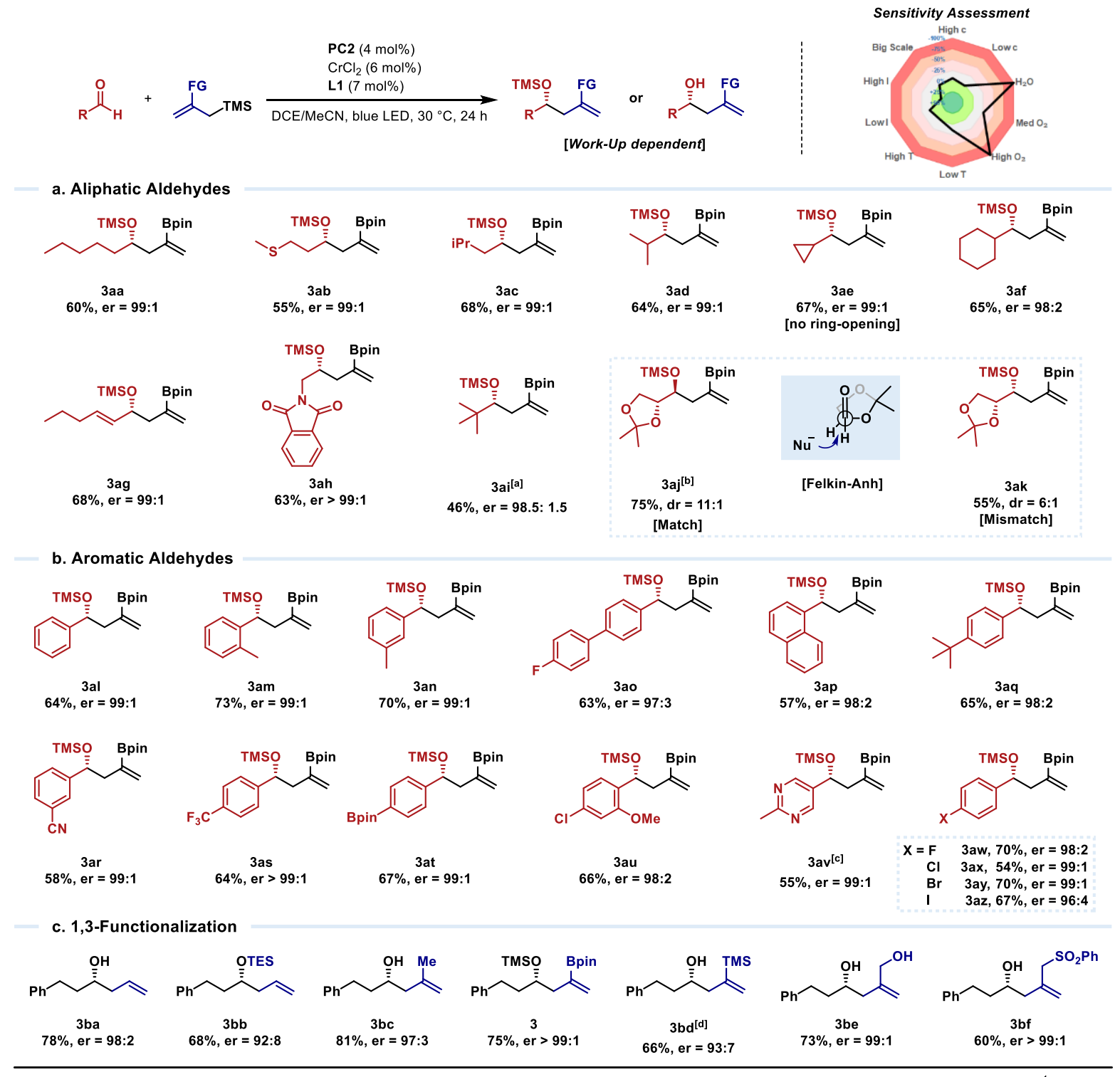

Standard conditions: Aldehyde $(0.2 \mathrm{mmol})$, allylsilane $(0.6 \mathrm{mmol}) .0 .1 \mathrm{M} . \lambda=425 \mathrm{~nm}$. Isolated yields reported. Bpin = Bis (pinacolato $)$ diboron. TMS $=$ Trimethylsilyl. [a] ${ }^{1} \mathrm{H}-\mathrm{NMR}$ yield. [b] Ligand ent ${ }^{-1}$ used. [c] Cat. loading doubled. [d] $T=0{ }^{\circ} \mathrm{C}$.

Figure 3. Substrate scope of the enantioselective allylation.

Aliphatic aldehydes showed great reactivity, including primary (3aa-3ac, 3ag, 3ah), secondary (3ad - 3af) and tertiary ones (3ai). The respective homoallylic alcohols could be obtained in good yields and excellent enantiomeric ratios. A thioether (3ab, 55\%, er = 99:1) as well as a phthalimide unit (3ah, $63 \%$, er > 99:1) were shown to be well tolerated. Cyclic and non-cyclic secondary aldehydes displayed equally high reactivity, as exemplary shown by isopropylaldehyde derived product 3 ad $(64 \%$, er $=99: 1)$ and cyclopropylcarbaldehyde derived compound 3ae $(67 \%$, er $=99: 1)$. No open-chain products were observed in the latter case (radical clock). Moreover an $\alpha, \beta$-unsaturated aldehyde could selectively be converted to the 1,2-addition product 3 ag $(68 \%$, er $=99: 1)$.

As a highlight, we tested which influence existing stereocenters exerted on the stereoinduction by reacting $\alpha$-chiral glyceraldehyde acetonide. Running the reaction with both enantiomers of ligand 1 , we 
were able to obtain both the anti- (3aj, $75 \%, d r=11: 1)$ and the syn-diastereomer $(3 a k, 55 \%, d r=6: 1)$ as separable main product respectively. This showed that the stereoinduction of the chiral chromium complex determines the stereochemical outcome and is able to override the Felkin-Anh type stereoinduction of the substrate. Although a classical "match" case for the anti- and a "mismatch" scenario for the syn-diastereomer was observed, this allowed the switchable synthesis of both diastereomers.

Next, our focus turned towards aromatic aldehydes. Benzaldehyde gave the respective product 3ai in good yield (64\%) and excellent enantioselectivity (er = 99:1). Investigating steric influences, it was shown that substitution in ortho (3am, 3au), meta (3an, 3ar) and para (3ao, 3aq, 3as - 3az) position were all well tolerated. Neither the sterically demanding tert-butyl substituent nor the presence of multiple substituents disturbed the reaction and the respective products $3 \mathrm{aq}(65 \%$, er $=98: 2)$ and 3 au $(66 \%$, er $=98: 2)$ could be successfully obtained. Investigating electronic influences, we were pleased that a variety of substituents were well tolerated, including cyano (3ar), trifluoromethyl (3as), methoxy (3au) or halide groups (3aw - 3az). Moreover, Bpin-substituted benzaldehyde gave product 3at (67\%, er $=99: 1$ ), bearing double boron substitution. Finally, pyrimidine carboxaldehyde gave the heterocylic homoallylic product 3 av $(55 \%$, er $=99: 1)$.

Eventually, the reactivity of different allylsilanes was investigated (Figure 3c). Allyltrimethylsilane showed the highest reactivity, giving free homoallylic alcohol $\mathbf{3 b a}(78 \%$, er $=98: 2)$ after deprotection. Allowing for orthogonal protecting group strategies, the conversion of allyl(triethyl)silane gave the more stable TES-protected product 3bb $(68 \%$, er $=92: 8)$. Different substituents in the vinylic position were very well tolerated, yielding the respective 1,3-functionalized alcohol products bearing for instance alkyl (3bc, 81\%, er =97:3), boron $(3,75 \%$, er $=99: 1)$ or silyl $(3 \mathbf{b d}, 66 \%$, er $=93: 7)$ moieties. As another highlight, the synthesis of diol 3 be $(73 \%$, er $=99: 1)$ or sulfonyl bearing alcohol 3 bf $(60 \%$, er $>99: 1)$ both proceeded in good yield and excellent selectivity, underlining the high functional group tolerance. 


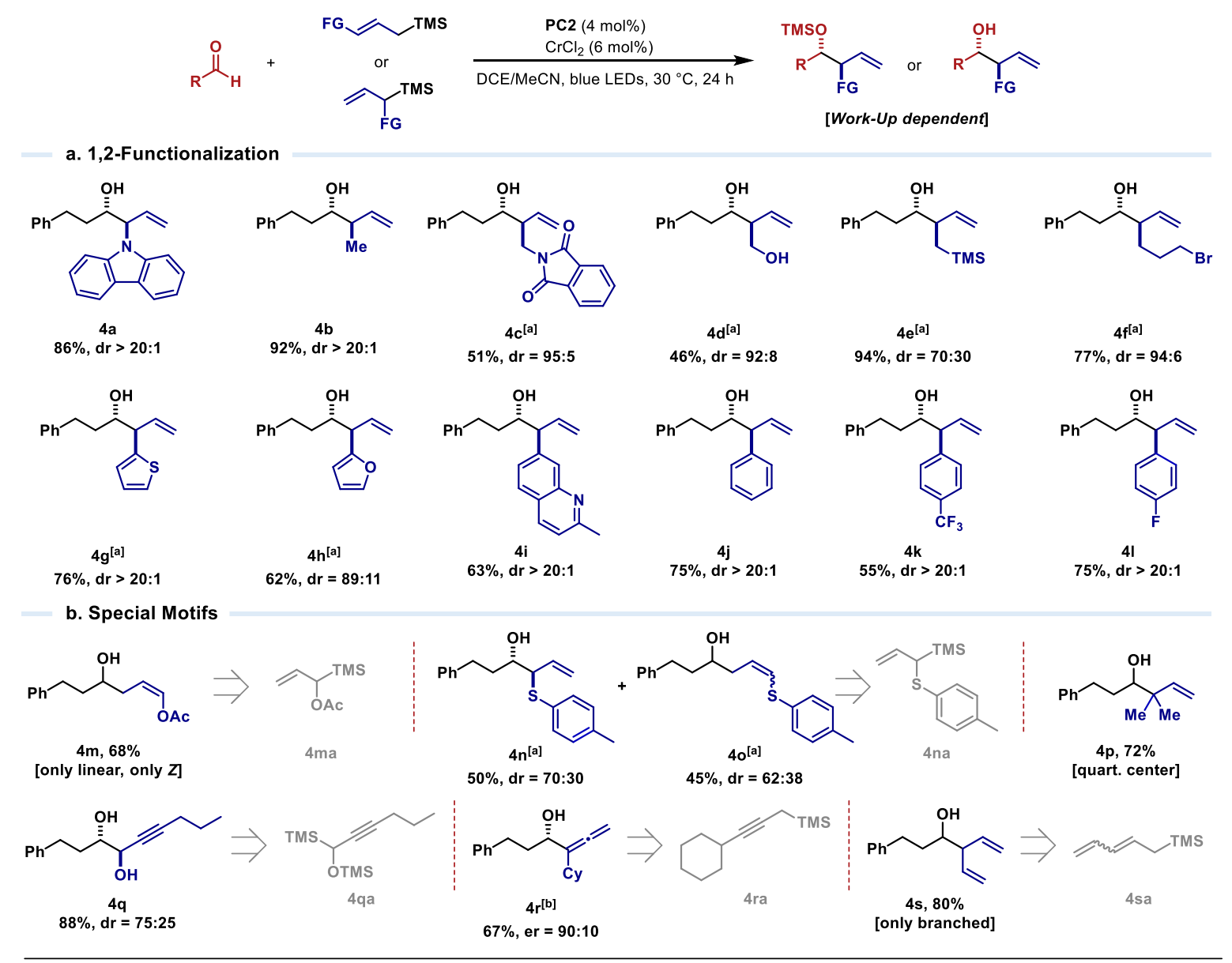

Standard conditions: Aldehyde $(0.2 \mathrm{mmol})$, allylsilane $(0.6 \mathrm{mmol}) .0 .1 \mathrm{M} . \lambda=425 \mathrm{~nm}$. Isolated yields reported TMS $=$ Trimethylsilyl. Cy $=$ Cyclohexyl. [a] Reaction run in THF/MeCN. [b] Conditions see Figure 3.

Figure 4. Substrate scope of the diastereoselective allylation.

Our method was also able to construct a variety of 1,2-functionalized homoallylic alcohols (Figure 4). However, if ligand 1 was added, the respective products were obtained in an average enantiomeric ratio below 90:10. This result shows that enantioinduction for these challenging substrates is feasible, but further ligand design will be conducted to improve the obtained selectivity. However, already the diastereoselective synthesis of these important structures is highly desirable, especially as we could access the respective anti-products with excellent diastereoselectivity while under Hosomi-Sakurai conditions the syn-diastereomers would be obtained.

Due to their relevance, these 1,2-homoallylic alcohols have been the target of dual chromium-catalyzed allylation protocols before. For example, our group reported the coupling between aldehydes and either allylamines $^{38}$, electron-rich allylarenes, silyl enol ethers ${ }^{43}$ or alkyl-substituted homoallylic species generated by alkyl radical addition to butadiene. ${ }^{40}$ In all of these cases, the substituent being introduced was also the center of activation and therefore automatically determined the scope. This led to several limitations, such as electron-neutral or -poor arenes, heteroarenes or non-alkyl-substituted homoallylic 
species. At the same time inspiring work by Kanai showed the direct oxidation of electron-rich alkenes ${ }^{39}$ as well as the HAT activation of unactivated alkenes. ${ }^{47}$ However, these methods focused on the important utilization of feedstock olefins and the synthesis of their respective aliphatic products.

Our approach of reacting substituted allylsilanes differs from previous methods in so far as that the high reactivity of our system benefits from the unique properties of the silane and is thus mostly independent from the employed substituents. Therefore, our protocol displays not only a general and flexible alternative to synthesize the product motifs being reported before, but more importantly it allows for the introduction of substituents which were previously inaccessible by dual chromium catalysis. The scope of 1,2-functionalized products is shown in Figure 4. Carbazole substituted allyltrimethylsilane gave access to the homoallylic aminoalcohol $4 a(86 \%, d r>20: 1)$ and methylallyltrimethylsilane gave the crotylated product $\mathbf{4 b}$ (92\%, dr > 20:1), both in excellent yield and selectivity. Also, allylbenzene product $4 \mathbf{j}$ could be obtained in $75 \%$ yield with $d r>20: 1$. Switching to unprecedented examples, the flexibility in regards of demanding substituents was tested. Fortunately, the thiophene, furane and quinoline containing products $\mathbf{4 g}-\mathbf{4 i}$ were obtained in high yields and with excellent levels of diastereoselectivity. Even challenging $p-\mathrm{CF}_{3}$ substituted allylbenzene was reactive and yielded compound $\mathbf{4 k}(55 \%$, $\mathrm{dr}>20: 1)$. Complementing the products of the alkyl radical addition to butadiene, the respective nitrogen, oxygen and silyl derivatives could be synthesized, affording 1,3-amino alcohol 4c, 1,3-diol 4d and 1,3-silyl alcohol $\mathbf{4 e}$.

Figure $4 \mathrm{~b}$ includes products, which stood out from the standard reactivity and show the bandwidth of reactive chromium intermediates reachable by our method. For example, acetate bearing allylsilane $\mathbf{4 m a}$ gave selective access to the linear homoaldol product $\mathbf{4 m}$, with only the $Z$-isomer being observed. This switch from branched to linear selectivity is highly interesting and can be explained by internal coordination between the acetate group and the chromium in the key intermediate, giving a stable fivemembered ring. ${ }^{48}$ Also, a challenging quaternary center could be generated, shown by the efficient synthesis of product $4 p(72 \%)$. To our delight not only allylsilanes, but also propargylsilanes showed reactivity under our conditions. When $\alpha$-OTMS-substituted propargyltrimethylsilane (4qa) was subjected to our reaction conditions, propargylic diol (4q) could be isolated in excellent yield. In contrast, if linear substituted propargyltrimethylsilane (4ra) was reacted, selectively the allenic alcohol (4r) was obtained. These results reveal how heavily the equilibrium between allenic and propargylic chromium intermediates is influenced by their substitution. ${ }^{49}$ Notably, our method thereby allows access to all three: 
allylic, allenic as well as propargylic alcohols. As a last highlight, the reaction of diene (4sa) solely yielded the branched product $\mathbf{4 s}$ in $80 \%$ yield. This is remarkable, as Pd or Ni typically give the linear product. 50

Application: The vinylboronic species generated by our method inhibited special reactivity, as the boron substituent can not only participate in a variety of transformations itself, but additionally activates the adjacent alkene. To showcase this unique utility, product 3 was subjected to four different functionalizations (Figure 5a)..$^{51}$
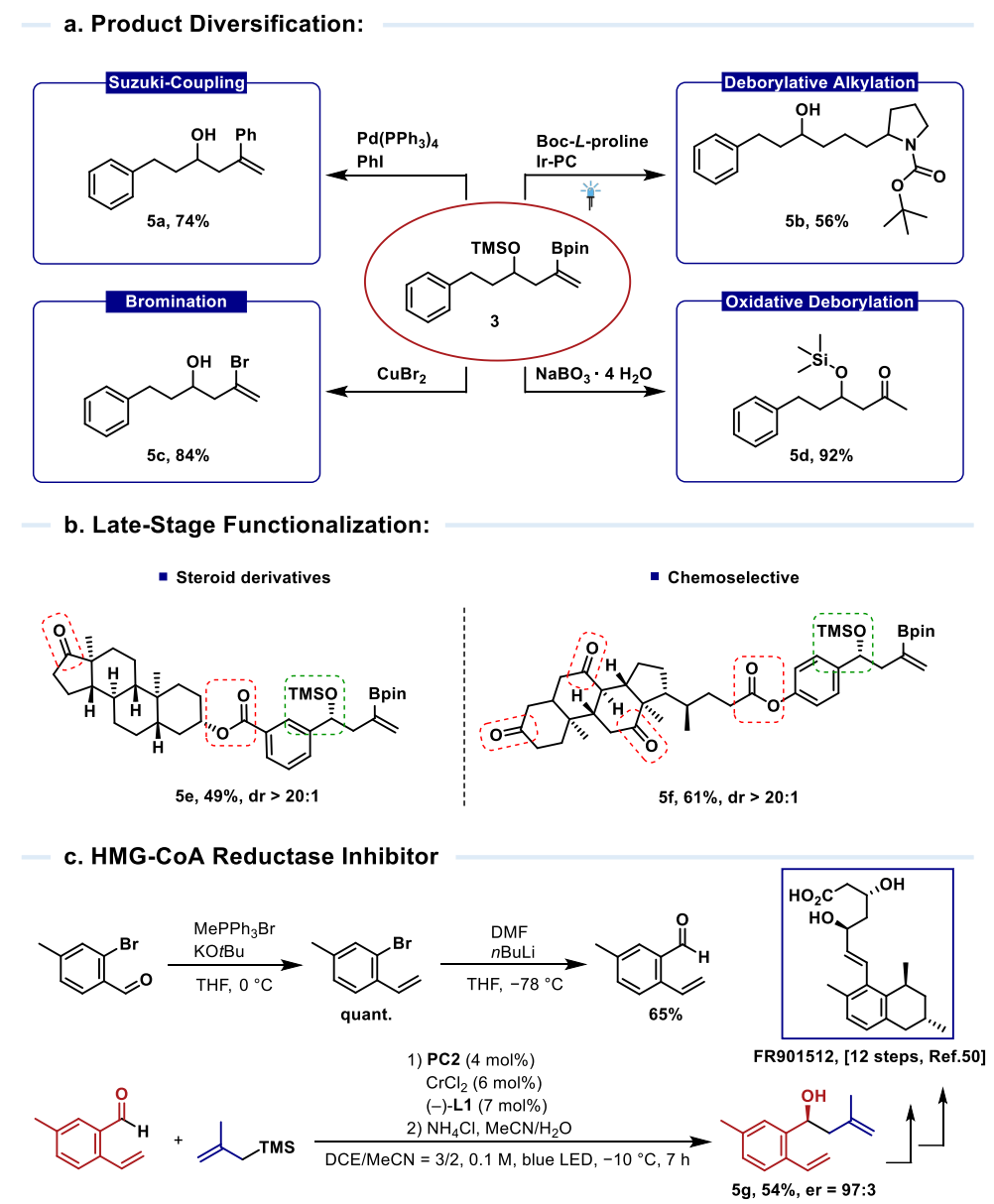

Figure 5. Different applications of our dual catalytic method.

First, Suzuki coupling with aryl iodide proceeded very smoothly, giving cross-coupled styrene 5 a in $74 \%$ yield. Next, deborylative radical addition to the linear position was shown, giving the alkylated product 5b. ${ }^{52}$ Finally, bromination with copper bromide afforded the vinylic halide $\mathbf{5 c}$ in very good yield $(84 \%)$ and oxidative deborylation enabled access to the enantioenriched $\beta$-hydroxyketone $\mathbf{5 d}$ in excellent yield of $92 \%$. Furthermore, the chemoselectivity of our method was showcased by its application in the latestage functionalization of complex, biologically relevant structures (Figure 5b). Therefore, two steroid derived aldehydes were chosen, bearing multiple electrophilic carbonyl moieties. Although this 
presented a significant challenge, reaction was solely observed at the aldehyde position, yielding the respective homoallylic products $\mathbf{5 e}$ and $\mathbf{5} \mathbf{f}$ in excellent diastereoselectivity. As a final application, building block $\mathbf{5 g}$ from the synthesis of the potent HMG-CoA Reductase Inhibitor FR90151253 was identified to be a possible target for our asymmetric protocol (Figure $5 \mathrm{c}$ ). While the preparation of the aldehyde starting material proceeded very smoothly, the enantioselective allylation key step however presented a significant challenge for two reasons. First, the styrene moiety overreacted under our stronglyoxidizing conditions and secondly, the steric demand diminished the enantioselectivity (er = 80:20). After short re-optimization it was found, that cooling to $-10^{\circ} \mathrm{C}$ in combination with a shorter reaction time cut down the overreaction and the product was obtained in $54 \%$ yield with a significantly improved er (97:3).

Mechanistic Investigation: The initial mechanistic rationalization started with the photocatalyzed SEToxidation of the allylsilane, followed by trapping of the generated allylic radical by $\mathrm{Cr}^{\prime \prime}$. Indeed, SternVolmer Quenching Studies (Figure 6b) confirmed that allyl species 2 exclusively quenches the excited photocatalyst and the addition of TEMPO as a radical scavenger inhibits product formation, but the TEMPO-trapped allylic intermediate could be isolated instead (Figure 6a). Moreover, the observed chemo-, diastereo- and enantioselectivity is in full agreement with the well reported reactivity of allylchromium species, adding to aldehydes via a selectivity determining Zimmerman-Traxler transition state.

If only a truly dual catalytic cycle was present, Crll' would be released from the alkoxide by $\mathrm{TMS}^{+}$and turned over by the reduced photocatalyst. Nevertheless, the high reaction rate challenged a careful mechanistic investigation and therefore, the quantum yields were measured (Figure 6c). The results (with ligand: $\Phi=6.8$; without ligand: $\Phi=15.8$ ) hint towards an additional chain mechanism being operative. To obtain better understanding about the efficiency of the putative chain reaction a 'light On/Off' experiment was conducted (Figure 6d). Interestingly, while throughout the first light off intervals the yield remained the same, starting from the fourth 'light off' interval, a yield increase between 3 and $5 \%$ was observed during the first halves of the respective dark periods. This indicates a chain mechanism which constantly has to be re-initiated to work efficiently and becomes more relevant at higher conversions. 

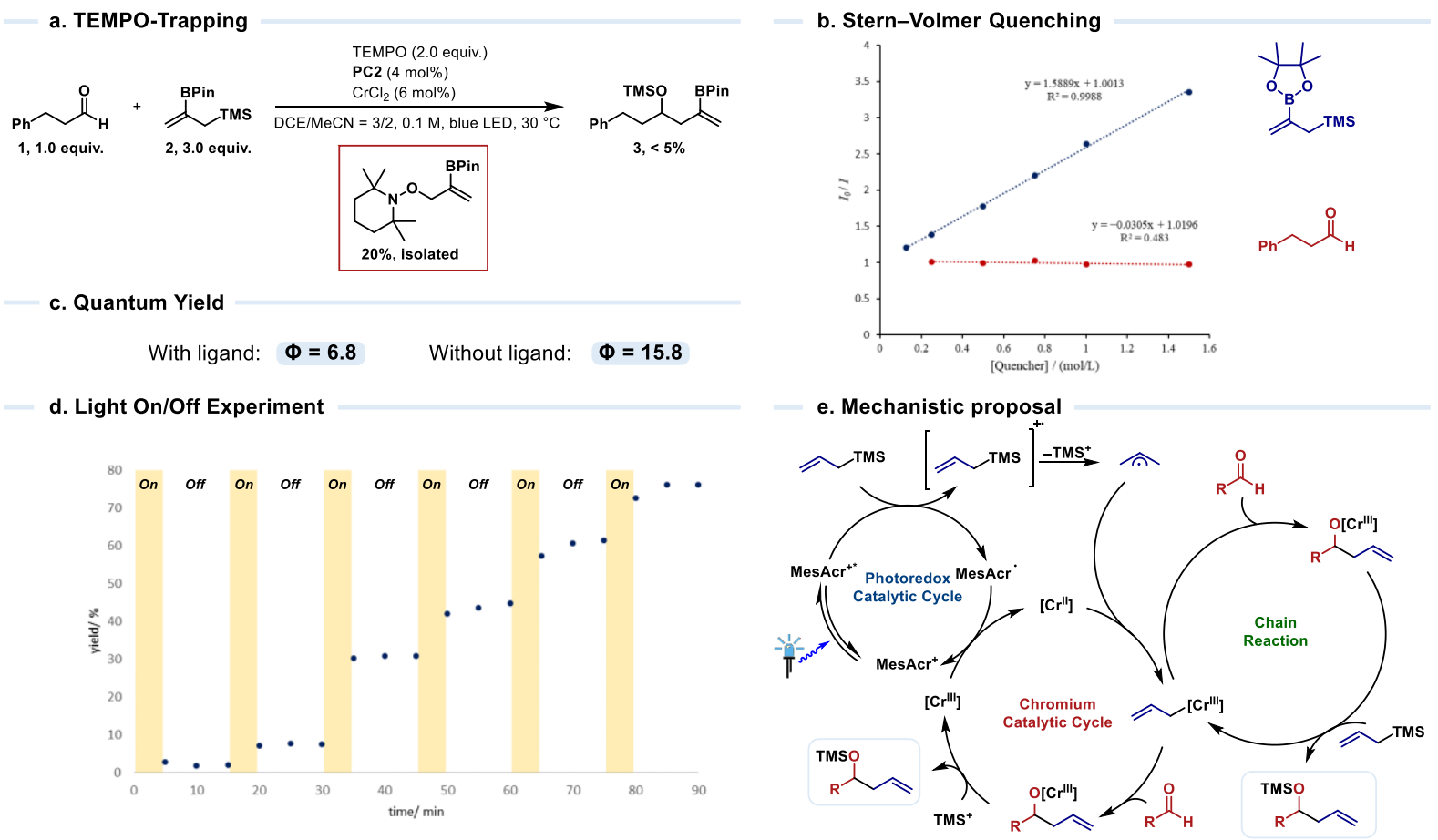

Figure 6. Mechanistic Investigation and resulting mechanistic proposal.

As the oxidative power of $\mathrm{Cr}^{\text {III }}\left(E_{1 / 2^{0 x}}=-0.51 \mathrm{~V} \text { vs SCE in DMF }\right)^{54}$ does not match the potential of allyltrimethylsilane $\left(E_{1 / 2}{ }^{\mathrm{ox}}=+1.54 \mathrm{~V}\right.$ in $\left.\mathrm{MeCN}\right),{ }^{44}$ a chain closure by a radical chain mechanism is thermodynamically disfavored. Instead, considering the high oxophilicity of silicon $\left(\mathrm{BDE}_{[\mathrm{Si}-\mathrm{O}]} \approx\right.$ $190 \mathrm{kcal} / \mathrm{mol}$ vs $\left.\mathrm{BDE}_{[\mathrm{Cr}-\mathrm{O}]} \approx 110 \mathrm{kcal} / \mathrm{mol}\right),{ }^{55}$ we propose a formal $\sigma$-bond metathesis taking place. In that case, after a first photocatalytic cycle, the chromium alkoxide would directly react with the allylsilane starting material to give the TMS-protected alcohol while generating another reactive allyl-chromium species. The putative metathesis between chromium alkoxides and silicon species has been discussed before, but was never subjected to a closer mechanistic look. ${ }^{10,41}$ Conceivable pathways would include either a concerted one, in which the chromium-bound alkoxide and an allylsilane form a four-membered transition state or alternatively a stepwise mechanism starting with the alkoxide dissociation first, followed by the attack from the allylsilane. To gain insight into the nature of this mechanism, DFT calculation was conducted (Figure 7, for methods used see SI). As expected, the selectivity-determining allylation was found to proceed via a Zimmerman-Traxler transition state (7d-TS), yielding chromium alkoxide $7 \mathrm{e}$. The following reactivity-determining [ $\mathrm{Cr}-\mathrm{O}]$-bond breakage was found to be likely mediated by an external $\mathrm{TMS}^{+}$, as for example generated by the photooxidation of the allylsilane. The respective transition state $(\mathbf{7 g}-\mathrm{TS})$, leads to the dissociation of the TMS-protected product $\mathbf{7 j}$ and leaves a cationic chromium-complex, which can easily be attacked by a second allylsilane, thus closing the chain in a 
stepwise manner. The transition state for the one-step concerted pathway was also found, but is energetically unfavored (energy barrier of more than $40 \mathrm{kcal} / \mathrm{mol}$, see SI).

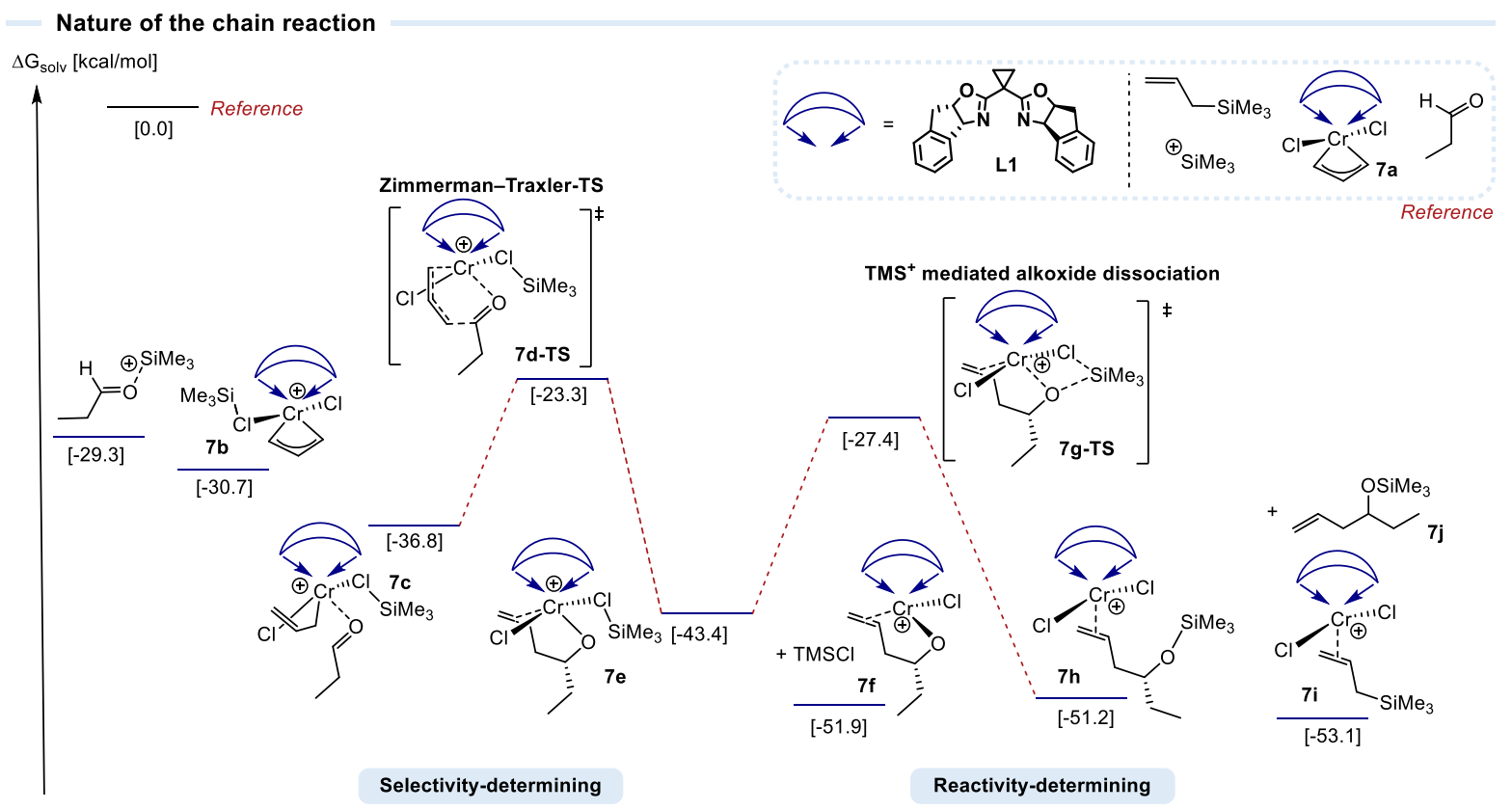

Figure 7. DFT-calculated, stepwise mechanism (PW6B95-D3/def2-TZVP//PBEh-3c+COSMO-RS).

Conclusion: The highly efficient, straightforward and enantioselective coupling of aldehydes with abundant allylsilanes is reported, leading to the formation of valuable chiral homoallylic alcohols. The complementary activation mechanism and the mild conditions of our dual catalytic design allowed us to synthesize a variety of these highly valuable products in opposite chemo- and diastereoselectivity to the established Hosomi-Sakurai conditions. Highlights of this work are the broad substrate scope showcasing high functional group tolerance, the excellent levels of enantioselectivity obtained and the focus on enantioenriched boron-substituted homoallylic alcohols. To emphasize the utility of our method, we reported different product diversifications, the late-stage functionalization of steroid derivatives and its application as the key step in the synthesis of a reductase inhibitor. Comprehensive mechanistical studies revealed that the reaction is likely to proceed mainly via a chain reaction mechanism, based on a formal $\sigma$-bond metathesis. As this mechanism has been proposed before, but was never carefully investigated, we elucidated the nature of this unprecedented chain reaction by DFT calculations and it was shown that the chain is likely to proceed via a TMS ${ }^{+}-$mediated, stepwise mechanism and not a concerted pathway. We believe that the generality and simplicity of our approach will render this method highly useful for the synthetic organic community and moreover, we hope that the mechanistical insight on the rather unusual chain mechanism will inspire new related activation strategies. 


\section{Acknowledgments}

We thank the Fonds der Chemischen Industrie (R. K., Kekule Scholarship No.: 106151), the Deutsche Forschungsgemeinschaft (Leibniz Award; S.D. and C.M.-L.: SFB 858) for generous financial support.

\section{Author Contributions}

$\S$ F.S. and S.D. contributed equally. All authors have given approval to the final version of the manuscript.

\section{Competing Interests}

The authors declare no competing interests.

\section{References}

1. Tietze, L. F., Kinzel, T. \& Brazel, C. C. The domino multicomponent allylation reaction for the stereoselective synthesis of homoallylic alcohols. Acc. Chem. Res. 42, 367-378 (2009).

2. Otera, J. (ed.). Modern Carbonyl Chemistry (Wiley-VCH Verlag GmbH, 2000).

3. Kaib, P. S. J., Schreyer, L., Lee, S., Properzi, R. \& List, B. Extremely Active Organocatalysts Enable a Highly Enantioselective Addition of Allyltrimethylsilane to Aldehydes. Angew. Chem. Int. Ed. 55, 13200-13203 (2016).

4. Yus, M., González-Gómez, J. C. \& Foubelo, F. Diastereoselective allylation of carbonyl compounds and imines: application to the synthesis of natural products. Chem. Rev. 113, 5595-5698 (2013).

5. Yamamoto, Y. \& Asao, N. Selective reactions using allylic metals. Chem. Rev. 93, 22072293 (1993).

6. Dutta, S., Bhattacharya, T., Werz, D. B. \& Maiti, D. Transition-metal-catalyzed C-H allylation reactions. Chem 7, 555-605 (2021).

7. Denmark, S. E. \& Fu, J. Catalytic enantioselective addition of allylic organometallic reagents to aldehydes and ketones. Chem. Rev. 103, 2763-2794 (2003).

8. Kim, I. S., Ngai, M.-Y. \& Krische, M. J. Enantioselective iridium-catalyzed carbonyl allylation from the alcohol or aldehyde oxidation level via transfer hydrogenative coupling of allyl 
acetate: departure from chirally modified allyl metal reagents in carbonyl addition. J. Am. Chem. Soc. 130, 14891-14899 (2008).

9. Kim, S. W., Zhang, W. \& Krische, M. J. Catalytic Enantioselective Carbonyl Allylation and Propargylation via Alcohol-Mediated Hydrogen Transfer: Merging the Chemistry of Grignard and Sabatier. Acc. Chem. Res. 50, 2371-2380 (2017).

10. Fürstner, A. \& Shi, N. Nozaki-Hiyama-Kishi Reactions Catalytic in Chromium. J. Am. Chem. Soc. 118, 12349-12357 (1996).

11. Tian, Q. \& Zhang, G. Recent Advances in the Asymmetric Nozaki-Hiyama-Kishi Reaction. Synthesis 48, 4038-4049 (2016).

12. Pulis, A. P., Yeung, K. \& Procter, D. J. Enantioselective copper catalysed, direct functionalisation of allenes via allyl copper intermediates. Chem. Sci. 8, 5240-5247 (2017).

13. Del Pozo, J. et al. Streamlined Catalytic Enantioselective Synthesis of $\alpha$-Substituted $\beta, \gamma^{-}$ Unsaturated Ketones and Either of the Corresponding Tertiary Homoallylic Alcohol Diastereomers. J. Am. Chem. Soc. 142, 18200-18212 (2020).

14. Meng, F., Jang, H., Jung, B. \& Hoveyda, A. H. Cu-catalyzed chemoselective preparation of 2-(pinacolato)boron-substituted allylcopper complexes and their in situ site-, diastereo-, and enantioselective additions to aldehydes and ketones. Angew. Chem. Int. Ed. 52, 5046$5051(2013)$.

15. Zhang, H., Huang, J. \& Meng, F. Cobalt-catalyzed diastereo- and enantioselective allyl addition to aldehydes and $\alpha$-ketoesters through allylic $\mathrm{C}-\mathrm{H}$ functionalization. Cell Rep. Phys. Sci. 2, 100406 (2021).

16. Chabaud, L., James, P. \& Landais, Y. Allylsilanes in Organic Synthesis - Recent Developments. Eur. J. Org. Chem., 3173-3199 (2004).

17. Sarkar, T. K. Methods for the Synthesis of Allylsilanes. Part 1. Synthesis, 969-983 (1990).

18. Sarkar, T. K. Methods for the Synthesis of Allylsilanes. Part 2. Synthesis, 1101-1111 (1990). 
19. Lambert, J. B. et al. The $\beta$ Effect of Silicon and Related Manifestations of $\sigma$ Conjugation. Acc. Chem. Res. 32, 183-190 (1999).

20. Hosomi, A. \& Sakurai, H. Syntheses of $\gamma, \delta$-unsaturated alcohols from allylsilanes and carbonyl compounds in the presence of titanium tetrachloride. Tetrahedron Lett. 17, 1295$1298(1976)$.

21. Hosomi, A. Characteristics in the reactions of allylsilanes and their applications to versatile synthetic equivalents. Acc. Chem. Res. 21, 200-206 (1988).

22. Hosomi, A., Shirahata, A. \& Sakurai, H. Chemistry of organosilicon compounds 113 chemoselective allylation of carbonyl compounds with allylsilanes promoted by tetra-butylammonium fluoride. A new synthesis of homoallyl alcohols. Tetrahedron Lett. 19, 3043-3046 (1978).

23. Pilcher, A. S. \& DeShong, P. Utilization of Tetrabutylammonium Triphenyldifluorosilicate as a Fluoride Source for Silicon-Carbon Bond Cleavage. J. Org. Chem. 61, 6901-6905 (1996).

24. Denmark, S. E. \& Almstead, N. G. Stereochemical Studies on the Addition of Allylsilanes to Aldehydes. The SE' Component. J. Org. Chem. 59, 5130-5132 (1994).

25. Chemler, S. R. \& Roush, W. R. In Modern Carbonyl Chemistry, edited by J. Otera, pp. 403490 (Wiley-VCH Verlag GmbH, 2000).

26. Lee, J. H. Use of the Hosomi-Sakurai allylation in natural product total synthesis. Tetrahedron 76, 131351 (2020).

27. Zhang, L. C., Sakurai, H. \& Kira, M. Enantioselective Allylation of Aldehydes Using Tartrate Ester Modified Allylsilanes. Chem. Lett. 26, 129-130 (1997).

28. Ishihara, K. et al. Catalytic asymmetric allylation using a chiral (acyloxy)borane complex as a versatile Lewis acid catalyst. J. Am. Chem. Soc. 115, 11490-11495 (1993).

29. Gauthier, D. R. \& Carreira, E. M. Catalytic, Enantioselective Addition of Allylsilanes to Aldehydes: Generation of a Novel, Reactive TilV Complex from TiF4. Angew. Chem. Int. Ed. 35, 2363-2365 (1996). 
30. Denmark, S. E. \& Fu, J. Catalytic, enantioselective addition of substituted allylic trichlorosilanes using a rationally-designed 2,2'-bispyrrolidine-based bisphosphoramide. $J$. Am. Chem. Soc. 123, 9488-9489 (2001).

31. Malkov, A. V. et al. Chiral 2,2'-bipyridine-type $\mathrm{N}$-monoxides as organocatalysts in the enantioselective allylation of aldehydes with allyltrichlorosilane. Org. Lett. 4, 1047-1049 (2002).

32. Malkov, A. V. et al. On the mechanism of asymmetric allylation of aldehydes with allyltrichlorosilanes catalyzed by QUINOX, a chiral isoquinoline N-oxide. J. Am. Chem. Soc. $130,5341-5348(2008)$.

33. Mahlau, M. \& List, B. Asymmetric counteranion-directed catalysis: concept, definition, and applications. Angew. Chem. Int. Ed. 52, 518-533 (2013).

34. Wadamoto, M. \& Yamamoto, H. Silver-catalyzed asymmetric Sakurai-Hosomi allylation of ketones. J. Am. Chem. Soc. 127, 14556-14557 (2005).

35. Yamasaki, S., Fujii, K., Wada, R., Kanai, M. \& Shibasaki, M. A general catalytic allylation using allyltrimethoxysilane. J. Am. Chem. Soc. 124, 6536-6537 (2002).

36. Prier, C. K., Rankic, D. A. \& MacMillan, D. W. C. Visible light photoredox catalysis with transition metal complexes: applications in organic synthesis. Chem. Rev. 113, 5322-5363 (2013).

37. Egorova, K. S. \& Ananikov, V. P. Toxicity of Metal Compounds: Knowledge and Myths. Organometallics 36, 4071-4090 (2017).

38. Schwarz, J. L., Schäfers, F., Tlahuext-Aca, A., Lückemeier, L. \& Glorius, F. Diastereoselective Allylation of Aldehydes by Dual Photoredox and Chromium Catalysis. J. Am. Chem. Soc. 140, 12705-12709 (2018).

39. Mitsunuma, H., Tanabe, S., Fuse, H., Ohkubo, K. \& Kanai, M. Catalytic asymmetric allylation of aldehydes with alkenes through allylic $\mathrm{C}(\mathrm{sp} 3)-\mathrm{H}$ functionalization mediated by organophotoredox and chiral chromium hybrid catalysis. Chem. Sci. 10, 3459-3465 (2019). 
40. Schwarz, J. L., Huang, H.-M., Paulisch, T. O. \& Glorius, F. Dialkylation of 1,3-Dienes by Dual Photoredox and Chromium Catalysis. ACS Catal. 10, 1621-1627 (2020).

41. Schwarz, J. L., Kleinmans, R., Paulisch, T. O. \& Glorius, F. 1,2-Amino Alcohols via Cr/Photoredox Dual-Catalyzed Addition of a-Amino Carbanion Equivalents to Carbonyls. J. Am. Chem. Soc. 142, 2168-2174 (2020).

42. Huang, H.-M., Bellotti, P., Daniliuc, C. G. \& Glorius, F. Radical Carbonyl Propargylation by Dual Catalysis. Angew. Chem. Int. Ed. 60, 2464-2471 (2021).

43. Schäfers, F. et al. Direct Access to Monoprotected Homoallylic 1,2-Diols via Dual Chromium/Photoredox Catalysis. ACS Catal. 10, 11841-11847 (2020).

44. Liu, R. et al. Visible-Light-Mediated Regioselective Allylation, Benzylation, and Silylation of Methylene-Malononitriles via Photoredox-Induced Radical Cation Fragmentation. Eur. J. Org. Chem., 1459-1465 (2020).

45. Pitzer, L., Sandfort, F., Strieth-Kalthoff, F. \& Glorius, F. Carbonyl-Olefin Cross-Metathesis Through a Visible-Light-Induced 1,3-Diol Formation and Fragmentation Sequence. Angew. Chem. Int. Ed. 57, 16219-16223 (2018).

46. Fyfe, J. W. \& Watson, A. J. Recent Developments in Organoboron Chemistry: Old Dogs, New Tricks. Chem 3, 31-55 (2017).

47. Tanabe, S., Mitsunuma, H. \& Kanai, M. Catalytic Allylation of Aldehydes Using Unactivated Alkenes. J. Am. Chem. Soc. 142, 12374-12381 (2020).

48. Kang, J. Y. \& Connell, B. T. Chromium-catalyzed homoaldol equivalent reaction employing a nucleophilic propenyl acetate. J. Am. Chem. Soc. 132, 7826-7827 (2010).

49. Fürstner, A. Carbon-Carbon Bond Formations Involving Organochromium(III) Reagents. Chem. Rev. 99, 991-1046 (1999).

50. Yang, J. et al. Efficient Palladium-Catalyzed Carbonylation of 1,3-Dienes: Selective Synthesis of Adipates and Other Aliphatic Diesters. Angew. Chem. Int. Ed. 60, 9527-9533 (2021). 
51. Wang, M., Gao, S. \& Chen, M. Stereoselective Syntheses of ( E)-ү',ס-Bisboryl-Substituted syn-Homoallylic Alcohols via Chemoselective Aldehyde Allylboration. Org. Lett. 21, 21512155 (2019).

52. Noble, A., Mega, R. S., Pflästerer, D., Myers, E. L. \& Aggarwal, V. K. Visible-Light-Mediated Decarboxylative Radical Additions to Vinyl Boronic Esters: Rapid Access to Y-Amino Boronic Esters. Angew. Chem. Int. Ed. 57, 2155-2159 (2018).

53. Inoue, M. \& Nakada, M. Structure elucidation and enantioselective total synthesis of the potent HMG-CoA reductase inhibitor FR901512 via catalytic asymmetric Nozaki-Hiyama reactions. J. Am. Chem. Soc. 129, 4164-4165 (2007).

54. Izutsu, K. Electrochemistry in nonaqueous solutions. 2nd ed. (Wiley-VCH, 2009).

55. Luo, Y.-R. Handbook of bond dissociation energies in organic compounds (CRC Press, 2003). 